\title{
A LARGE AREA SEARCH FOR RADIO-LOUD QUASARS WITHIN THE EPOCH OF REIONIZATION
}

\author{
M.J. JARVIS ${ }^{1}$, S. RAWLINGS ${ }^{1}$, F.E. BARRIO ${ }^{1}$, \\ G.J. HILL ${ }^{2}$, A. BAUER ${ }^{2}$, S. CROFT ${ }^{3}$ \\ ${ }^{1}$ Astrophysics, Department of Physics, Keble Road, Oxford, OX1 3RH, UK. \\ ${ }^{2}$ McDonald Observatory, University of Texas at Austin, 1 University Station \\ C1402, Austin, TX 78712-0259, USA. ${ }^{3}$ IGPP, Lawrence Livermore National \\ Laboratory, L-413, 7000 East Ave., Livermore, CA 94550, USA \\ E-mail:mjj@astro.ox.ac.uk
}

\begin{abstract}
The Universe became fully reionized, and observable optically, at a time corresponding to redshift $z \sim 6.5$, so it is only by studying the HI and molecular absorption lines against higher-redshift, radio-loud sources that one can hope to make detailed studies of the earliest stages of galaxy formation. At present no targets for such studies are known. In these proceedings we describe a survey which is underway to find radio-loud quasars at $z>6.5$, and present broad-band SEDs of our most promising candidates.
\end{abstract}

\section{Introduction}

The epoch of reionization has now been discovered as a protracted period reaching from $z \sim 20 \rightarrow 6.5^{1,2}$. However, prior to $z \sim 6.5$ galaxy formation was already well underway (e.g. [3]). It is essentially impossible to study this 'grey age' at optical wavelengths, but great progress can be expected if radio and millimetre telescopes can be targeted on quasars observed within the reionization epoch.

Radio-loud targets allow absorption studies that can probe the evolving neutral and molecular content of the high- $z$ Universe ${ }^{4}$, and radio HI absorption is the only way of probing the neutral gas which goes on to form stars. We could begin these studies with current facilities (e.g. the GBT and GMRT), and with the next generation of large radio telescopes, such as the LOFAR and the SKA, we will easily be able reach depths of lower luminosity radio sources and still detect $21 \mathrm{~cm}$ absorption. Unfortunately, there are currently no known $z>6.5$ radio-loud objects.

This is because such objects are rare, $\ll 1$ per cent of the radio population. Interest in pursuing them was dampened by the claim of a much 
sharper cut-off in their redshift distribution ${ }^{5}$ than earlier work ${ }^{6}$ had suggested. Jarvis \& Rawlings ${ }^{7}$ and Jarvis et al. ${ }^{8}$ have re-examined all the evidence concerning this redshift cutoff, obtaining results strongly favouring a fairly gradual decline with redshift.

\section{Design of the survey}

Jarvis \& Rawlings emphasized the care needed in sample selection and analysis. Therefore, the survey is selected at low frequency to avoid losing the highest- $z$ quasars, because of the steepening of radio spectra at high rest-frame frequencies. The only low-frequency $(325 \mathrm{MHz})$ survey with the required depth and sky coverage is WENSS/WISH ${ }^{10,11}$.

The sky-area and further information about the survey can be found in Jarvis et al. ${ }^{9}$. To summarize, there are approximately 10000 sources over an area of $\sim 1$ quarter of the sky.

\section{Eliminating low-redshift radio sources}

There is a challenging, but tractable, sifting problem to eliminate both galaxies and quasars at $z<6$. This is done in four steps.

(i) We have cross-correlated the radio sample with publicly available all-sky optical and near-IR imaging, i.e. SDSS, POSS and 2MASS, along with more general searches of known objects via the literature and NED. From this investigation we have optical IDs for about $67 \%$ of the objects in the northern sample (comprising quasars, low-redshift galaxies, BL Lac objects etc.). The remaining objects have no detectable optical emission, typically to $R \sim 21.5$.

(ii) We have initiated deeper targeted observations in R-band of the remaining sources in the northern hemisphere, with IGI at the $2.7 \mathrm{~m}$ at McDonald Observatory. Observations down to $R \sim 23-23.5$ (depending on conditions) have shown that we again cut the source list down by approximately 65 per cent.

These sources, generally extended objects which are presumably $z \lesssim 2$ radio galaxies, are obviously not at $z>6.5$, because like the $z \sim 6.5$ quasars already known ${ }^{12}$, these must have zero flux below the redshifted Lyman limit due to the Gunn-Peterson trough.

(iii) We use good-seeing near-IR imaging to find all the remaining quasars, and eliminate all the remaining galaxies. This part of the survey has been underway since August 2003 for the northern hemisphere, with a large allocation of time on the United Kingdom Infrared Telescope 
(UKIRT). This is now 75 per cent complete with the other 25 per cent set to be completed by August 2004.

(iv) We take Z-band or near-IR spectra and find the $z>6.5$ quasars. UKIRT-UIST has recently showed its capability in detecting quasar broademission lines in the highest redshift quasar known to date ${ }^{13}$ allowing an estimate of the black-hole mass in this quasar via the broad MgII emission line. Lyman- $\alpha$ is more than twice as bright as MgII in the composite SDSS quasar spectrum and the huge drop blueward of Lyman- $\alpha$ due to absorption by neutral hydrogen is also a very strong signature. Therefore, identification would be relatively easy in $\sim 20$ min exposures on the Hobby-Eberly telescope with its planned J-band extension to its low-resolution spectrograph. We have also been granted time on the Gemini-North telescope to do Z-band spectroscopy of our highest priority candidates.

The southern survey will commence in April 2004 using the ESO telescopes in Chile.

\section{4. $z>6$ radio-loud quasar candidates}

It is already clear that our best candidates come in two flavours (Fig 1.). Flavour-1 are 'textbook candidates' (top panel) with smooth JHK spectral energy distributions (SEDs): although photometry gives only a crude estimate, it seems very likely that these are quasars at redshifts of at least 6 (to explain the sharp break between I and J). Flavour-2 are 'bumpy-SED' objects (bottom panel) for which the only explanation we can find is that they are lightly-reddened quasars at $z \sim 2.5$. As the errors show, we cannot, however, rule out the possibility that these too are at $z>6$, perhaps with some reddening, so each of these must be followed up spectroscopically too.

To date we have six good $z>6$ quasar candidates, follow-up spectroscopy of these in the near future will provide the information on their true nature, and hopefully provide us with the discovery of the first $z>6$ radio-loud quasars.

Discovery of such objects will lead to the first $21 \mathrm{~cm}$ absorption observations within the epoch of reionization with the new generation of radio telescopes operating at frequencies of $\nu<300 \mathrm{MHz}$, e.g. the LOFAR.

\section{References}

1. A. Kogut, et al., ApJS, 148, 161 (2003).

2. R.L. Becker, et al., AJ, 122, 2850 (2001). 

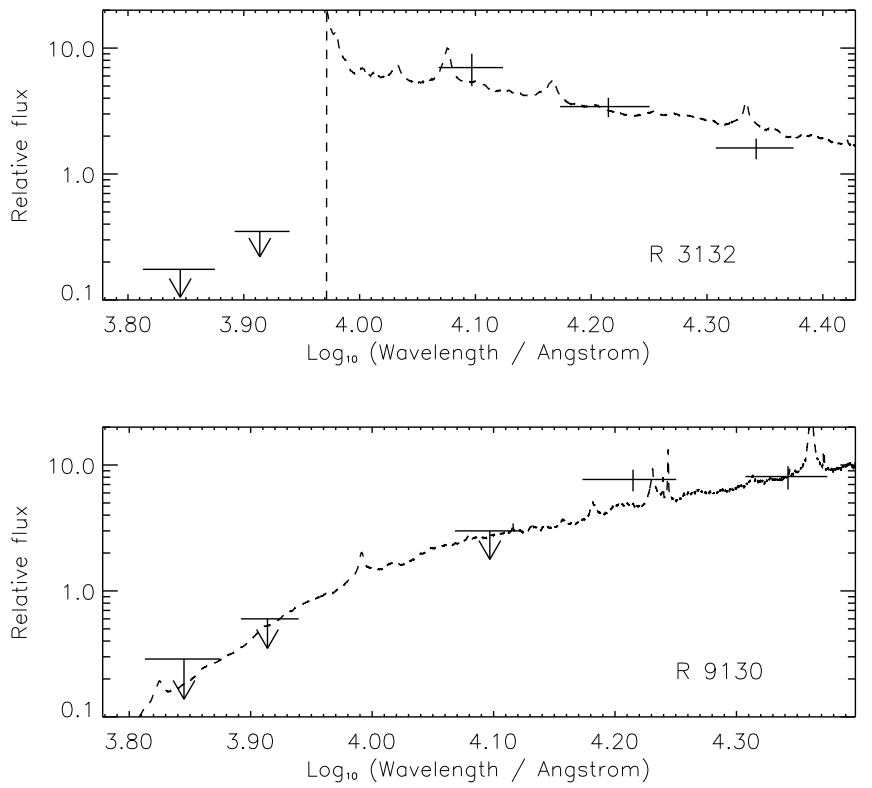

Figure 1. SEDs for two of our best candidates, the dashed line is the SDSS composite quasar spectrum. (top) The quasar composite is redshifted to $z=6.7$, and the solid horizontal lines are our photometric data points from our UKIRT observations. This is the 'textbook' SED of a $z>6.5$ quasar. (bottom) A reddened $\left(\mathrm{A}_{v}=1\right)$ quasar composite redshifted to $z=2.5$. This is our typical flavour-2 candidate.

3. R. Pello, D. Schaerer, J. Richard, J.-F. Le Borgne, J.-P. Kneib, $A \mathscr{E} A$, 416, L35 (2004).

4. C.L. Carilli, N.Y. Gnedin, F. Owen, ApJ, 577, 22 (2002).

5. P.A. Shaver, et al., Nature, 384, 439 (1998).

6. J.S. Dunlop, Peacock J.A., MNRAS, 247, 19 (1990).

7. M.J. Jarvis, S. Rawlings, MNRAS 319, 121 (2000).

8. M.J. Jarvis, et al., MNRAS, 327, 907 (2001).

9. M.J. Jarvis, et al., To appear in "AGN Physics with the Sloan Digital Sky Survey", ed. G. T. Richards and P. B. Hall (San Francisco: ASP), astroph/0309379, (2004)

10. R.B. Rengelink, et al., $A \& A S, 124,259$ (1997).

11. C. De Breuck, et al., $A \& A, 394,59$ (2002).

12. X. Fan et al., AJ, 125, 1649 (2003).

13. C.J. Willott, R.J. McLure, M.J. Jarvis, ApJ, 587, L15 (2003) 